\title{
Mitochondrial genotype modulates mtDNA copy number and organismal phenotype in Drosophila
}

\author{
Tiina S. Salminen a,1, Marcos T. Oliveira a,b,c,1 ${ }^{a}$ Giuseppe Cannino ${ }^{\mathrm{a}}$, Päivi Lillsunde ${ }^{\mathrm{a}}$, \\ Howard T. Jacobs ${ }^{\text {a,d }}$, Laurie S. Kaguni ${ }^{\mathrm{a}, \mathrm{b}, *}$ \\ a Institute of Biosciences and Medical Technology, FI-331014, University of Tampere, Finland \\ b Department of Biochemistry and Molecular Biology, Center for Mitochondrial Science and Medicine, Michigan State University, East Lansing, MI 48824, USA \\ c Departamento de Tecnologia, Faculdade de Ciências Agrárias e Veterinárias, Universidade Estadual Paulista “Júlio de Mesquita Filho", Jaboticabal, SP 14884-900, Brazil \\ ${ }^{\mathrm{d}}$ Institute of Biotechnology, FI-00014, University of Helsinki, Helsinki, Finland
}

\section{A R T I C L E I N F O}

\section{Article history:}

Received 23 June 2016

Received in revised form 28 November 2016

Accepted 1 February 2017

Available online 16 February 2017

\section{Keywords:}

Mitochondrial DNA

Haplotype

Cybrid

Respiration

Supercomplexes

\begin{abstract}
A B S T R A C T
We evaluated the role of natural mitochondrial DNA (mtDNA) variation on mtDNA copy number, biochemical features and life history traits in Drosophila cybrid strains. We demonstrate the effects of both coding region and non-coding $\mathrm{A}+\mathrm{T}$ region variation on mtDNA copy number, and demonstrate that copy number correlates with mitochondrial biochemistry and metabolically important traits such as development time. For example, high mtDNA copy number correlates with longer development times. Our findings support the hypothesis that mtDNA copy number is modulated by mtDNA genome variation and suggest that it affects OXPHOS efficiency through changes in the organization of the respiratory membrane complexes to influence organismal phenotype.
\end{abstract} (c) 2017 Elsevier B.V. and Mitochondria Research Society. All rights reserved.

\section{Introduction}

Of all processes that transpire in animal mitochondria, the production of ATP via oxidative phosphorylation (OXPHOS) is unique in the sense that the enzymatic complexes that perform electron transfer and ATP synthesis are products of a combination of polypeptides encoded by two distinct genomes. Thirteen of these polypeptides are encoded by the mitochondrial DNA (mtDNA), whereas the remainder of the OXPHOS-related proteins are encoded in nuclear DNA (nDNA), as are $\sim 1500$ additional proteins required for other mitochondrial functions. Hence, it is required that mitochondria maintain a coordinated communication with the nucleus via a series of anterograde and retrograde signaling processes for proper assembly and function of the OXPHOS complexes. Different combinations of mtDNA- and nDNAencoded OXPHOS gene variants might alter this signaling and/or OXPHOS assembly/function, causing changes at the phenotypic level that may be under selective pressure. Thus, it is expected that the most fit mitochondria have co-evolved mtDNA- and nDNA-encoded gene products that reflect inter-genomic epistatic interactions leading to an optimum level of OXPHOS performance and consequently, to

\footnotetext{
* Corresponding author at: Department of Biochemistry and Molecular Biology, Michigan State University, East Lansing, MI 48824, USA.

E-mail address: lskaguni@msu.edu (L.S. Kaguni).

1 These authors contributed equally to this work
}

appropriate organismal phenotypes for a particular environment. In fact, altered phenotypes caused by a putative disruption of co-evolved nuclear-mitochondrial OXPHOS function have been shown for different traits and organisms (Ballard and Whitlock, 2004; Dowling et al., 2007). In addition, naturally occurring mtDNA variants have been shown to affect OXPHOS biochemistry (Katewa and Ballard, 2007; Wolff et al., 2016), production of reactive oxygen species (Moreno-Loshuertos et al., 2006), and other aspects of cellular (Kazuno et al., 2006) and organismal physiology (Ballard et al., 2007).

mtDNA differs from the nuclear genome in several aspects: it is maternally inherited, contains no introns and very few non-coding intergenic nucleotides, possesses a single large non-coding region in most animal species and uses a variant genetic code. mtDNA replication occurs independently of nuclear DNA replication, although all trans-acting factors associated with the replication process are encoded by nDNA (Bogenhagen and Clayton, 1977). mtDNA copy number varies according to the energy requirements of tissues (Taylor and Turnbull, 2005), ranging from 2 to 10 copies per mitochondrion, and from hundreds to thousands of copies per cell in energy-demanding tissues. In Drosophila melanogaster, the single mtDNA non-coding region is called the $\mathrm{A}+\mathrm{T}$ region because of its high content of deoxyadenylate and thymidylate residues (90-96\%) (Fauron and Wolstenholme, 1976), and experimental evidence suggests it contains the origin of mtDNA replication (Goddard and Wolstenholme, 1978; Jõers and Jacobs, 2013). The A+T region is divided in two sub-regions, each with distinct, tandemly- 
repeated DNA sequence elements, plus non-repetitive intervening and flanking regions that comprise about $22 \%$ of its length (Lewis et al., 1994). The repeat I region carries a variable number of repeats of 338-373 bp in size (Lewis et al., 1994; Chen et al., 2012), whereas the repeat II region consists of four intact $464 \mathrm{bp}$ repeats and a fifth partial repeat of 137 bp (Lewis et al., 1994). Sequence variation within the $\mathrm{A}+\mathrm{T}$ region may be particularly important, because this region may contain the main regulatory elements for both mtDNA replication and gene expression (Goddard and Wolstenholme, 1978; Jõers and Jacobs, 2013). However, how naturally occurring mtDNA sequence variation in the $\mathrm{A}+\mathrm{T}$ and coding regions influences mtDNA copy number in ways systematically related to organismal phenotype has not been studied extensively, although this would serve to enhance our understanding of the role of the mitochondrial genome in metabolic regulation.

In addition to the coordinated assembly of nuclear and mitochondrial-encoded proteins into four OXPHOS complexes, these complexes can be further organized into dynamic supramolecular structures called supercomplexes (SCs) (Acín-Pérez et al., 2008). Recent findings suggest that short-term regulation of OXPHOS activity occurs at the level of SCS within the inner mitochondrial membrane, with a direct impact on cellular metabolism (Cogliati et al., 2016). The optimal function and abundance of SCs in relation to different stimuli is to enhance electron transport chain efficiency and ultimately cellular respiration. Variation in SC formation can be caused by varying external and internal factors, such as in disease states (Rosca et al., 2008). SCs have been found throughout eukaryotes (Chaban et al., 2014), and their reorganization in response to different stimuli may be considered a plastic response of OXPHOS function in changing conditions. To our knowledge, the impact that different mtDNA variants have on SC formation has not been studied to date.

Here, we characterize the sequence and length variation in the mtDNA coding and $A+T$ regions of ten naturally occurring haplotypes of $D$. melanogaster, and investigate the effects of these haplotypes in combination with their original and controlled nuclear backgrounds. Our hypothesis was that the mtDNA variation among these lines could be reflected in metabolically dependent phenotypes such as development time and body weight via different biochemical properties, including mtDNA copy number, oxygen consumption and SC formation and activity. We found a striking positive correlation between mtDNA copy number and development time, and suggest that this occurs because of increased oxygen consumption caused by higher abundance of OXPHOS SCs in lines with lower mtDNA copy number. Our study contributes to understanding the effects of mito-nuclear genome combinations on mitochondrial function and how this could be related to important organismal phenotypes.

\section{Materials and methods}

\subsection{Fly strains, backcrossing and rearing conditions}

Ten wild-type $D$. melanogaster strains, selected according to their geographic origin (Table S1), were obtained from Bloomington Drosophila Stock Center and backcrossed over ten generations, so as to contain the nuclear background of the Oregon RT strain (Oregon R strain maintained long-term in Tampere, Finland; ORT) combined with each of the unique mtDNA haplotypes. Backcrossing, as widely-used in mitochondrial research to obtain specific mtDNA haplotypes in controlled nuclear backgrounds, was conducted using males from the nucleardonor strain and females carrying mtDNA from another (Chen et al., 2012; Hutter and Rand, 1995; James and Ballard, 2003). Four selected mtDNA haplotypes (ORT, KSA2, WT5A and VAG1) were also backcrossed into the wild-type M2 nuclear background by the same procedure. M2 was selected as a nuclear background because its mtDNA possesses the highest number of unique nucleotide substitutions among all variants (see Section 3.1). The absence of the endosymbiotic Wolbachia was confirmed by PCR using Wolbachia-specific primers (Chen et al., 2012). Flies were cultured on standard medium (Toivonen et al., 2001) at $25{ }^{\circ} \mathrm{C}$, with $12 \mathrm{~h}$ periods of light and darkness and $60 \%$ humidity.

\subsection{Extraction of total DNA, mitochondria and mitochondrial DNA}

Total DNA was extracted using the phenol-chloroform-isoamyl alcohol method (Chen et al., 2012) from pools of 10 flies of a given strain, sex and age. For $\mathrm{A}+\mathrm{T}$ region sequencing, mtDNA was extracted from isolated mitochondria of the strains in their original nuclear background. Batches of 50-200 flies were homogenized at $4^{\circ}$ with eight strokes of a Teflon pestle in a glass homogenizer, in $4 \mathrm{ml} \mathrm{HB}$ medium $(225 \mathrm{mM}$ mannitol, $75 \mathrm{mM}$ sucrose, $1 \mathrm{mM}$ EDTA, 0.1\% BSA and $10 \mathrm{mM}$ Tris- $\mathrm{HCl}$, $\mathrm{pH}$ 7.6). The homogenate was transferred to a $15 \mathrm{ml}$ plastic centrifuge tube, filled with $\mathrm{HB}$ and then centrifuged for $5 \mathrm{~min}$ at $1000 g_{\max }$ at $4^{\circ}$ to pellet cell debris and nuclei. The supernatant was decanted to a new $15 \mathrm{ml}$ tube and crude mitochondria were pelleted for $10 \mathrm{~min}$ at $12,000 g_{\max }$ at $4^{\circ}$. The pellet was washed once, then resuspended in $300 \mu \mathrm{HB}$ and overlaid onto a $1.5 \mathrm{M} / 1 \mathrm{M}$ sucrose $(2+2 \mathrm{ml})$ step gradient and centrifuged for $1 \mathrm{~h}$ at $180,000 g_{\max }$ at $4^{\circ}$. The mitochondrial layer was transferred to a $2 \mathrm{ml}$ tube, and one volume of HB was added. Mitochondria were pelleted for $5 \mathrm{~min}$ at $12,000 \mathrm{~g}_{\max }$ at $4^{\circ}$, then processed for mtDNA extraction using the phenol-chloroform-isoamyl alcohol method (Chen et al., 2012).

\section{3. $m t D N A$ sequencing and sequence analyses}

The coding region of the mitochondrial genomes was amplified, as previously (Chen et al., 2012), in three overlapping fragments (of $\sim 5.5 \mathrm{~kb}$ in size) by long-PCR (LongRange PCR kit, Qiagen), using total DNA as template. The PCR conditions were: $93^{\circ} \mathrm{C}$ for $3 \mathrm{~min}$, followed by 35 cycles of $93{ }^{\circ} \mathrm{C}$ for $15 \mathrm{~s}, 50{ }^{\circ} \mathrm{C}$ for $30 \mathrm{~s}$, and $60{ }^{\circ} \mathrm{C}$ for $6 \mathrm{~min}$, with a final step of $60^{\circ} \mathrm{C}$ for $10 \mathrm{~min}$. PCR products were verified by $1 \%$ $(\mathrm{w} / \mathrm{v})$ agarose gel electrophoresis, gel-purified and used as templates for the sequencing reactions, using primers as described by Chen et al. (Chen et al., 2012). Both strands of the mtDNA fragments were entirely sequenced using the Big Dye v3.1 kit and a 3130xl genetic analyzer (Applied Biosystems). The sequences were analyzed and the coding region was assembled using the phred/phrap/consed package (Gordon et al., 1998). Deviations from the reference sequence were confirmed by a second PCR and sequencing of the amplified fragment. To confirm that the backcrossing procedure was successful and that each of the cybrid strains contained the expected mtDNA haplotype, specific mtDNA regions from each of the backcrossed cybrids were amplified and sequenced. The entire sequence of the ten mtDNA coding regions and that of Drosophila simulans (used as an outgroup, GenBank CM002915.1) was aligned using Clustal Omega, and the alignment was inputted in the TreeFinder program (Jobb et al., 2004) for inference of the maximum likelihood phylogenetic tree, with 1000 bootstrap replicates. Methods for the $\mathrm{A}+\mathrm{T}$ region sequencing (and mapping of the repeat region length) are described in the Supplementary File.

\subsection{Relative mtDNA copy number determination}

We measured relative mtDNA copy number levels from two-hour, and three-, ten- and 25-day old virgin females and males, and from pupae from strains in their original nuclear background and as ORT cybrids. To do so, $50 \mathrm{ng}$ of total DNA was used as template in quantitative PCR using primers for the mitochondrial 16S rRNA gene (16S-R 5'TCGTCCAACCATTCATTCCA-3' and 16S-L 5'-TGGCCGCAGTATTTTGACTG$\left.3^{\prime}\right)$ and the nuclear RpL32 gene (RpL32F 5'-AGGCCCAAGATCGTGAA GAA-3' and RpL32A 5'-TGTGCACCAGGAACTTCTTGAA-3'). Reactions were performed in a StepOnePlus instrument (Applied Biosystems) using the Fast SYBR Green Master Mix (Applied Biosystems) under the manufacturer's recommended conditions: $95^{\circ} \mathrm{C}$ for $20 \mathrm{~s}$, followed by 
40 cycles of $95^{\circ}$ for $3 \mathrm{~s}$ and $60^{\circ}$ for $30 \mathrm{~s}$. Calibration curves were used to quantitate mtDNA copy numbers relative to nDNA, based on the linear relationship between the crossing point-cycle values and the logarithm of the starting copy number. The average $C_{T}$ and SD were calculated from three technical replicates per sample, from four biological replicates. The $\Delta \Delta \mathrm{C}_{\mathrm{T}}$ values were calculated by comparing the ratios between the nuclear and mitochondrial target genes. The relative mtDNA copy number values were derived by comparing each of the sex, strain and age group samples to the same internal control sample that was arbitrarily given the value 1.0. The internal control sample for the experiments shown in Figs. 2, 3, S3 and S4 consisted of pooled total DNA from 200 ORT males of 25 days or older, whereas the one shown in Figs. 4 and S7 consisted of pooled total DNA from 200 ORT males of one-to-five days of age.

\subsection{Measurements of egg-to-adult development time and juvenile body weight}

Four vials containing 20 pre-mated females and 10 males per vial were used for egg laying on four consecutive days (5-8 h daily). Vials containing $>100$ eggs were excluded from the analysis. Time of development was determined by counting the number of females and males enclosed on a particular day (vials checked once a day) and comparing it to the day of egg laying. Within one day after eclosion, the juvenile body weight of females and males was measured in pools of three individuals, using a calibrated analytical balance and 20-30 biological replicates per strain and sex.

\subsection{Oxygen consumption measurements}

Total protein lysate was prepared from batches of $12-15$ pupae or three day-old flies from four selected ORT cybrid strains (haplotypes ORT, KSA2, WT5A and VAG1). Pupae or adult flies were homogenized gently with a mortar and pestle at $4{ }^{\circ} \mathrm{C}$ in $500 \mu \mathrm{l}$ of cold isolation buffer (250 mM sucrose, 5 mM Tris-HCl, 2 mM EGTA, pH 7.4) and then filtered through muslin. Oxygen consumption was measured with a Clarke-type oxygen electrode (Oxygraph, Hansatech Instruments Ltd., Norfolk, UK) in assay buffer ( $120 \mathrm{mM} \mathrm{KCl}, 5 \mathrm{mM} \mathrm{KH_{2 }} \mathrm{PO}_{4}, 3 \mathrm{mM}$ HEPES-KOH, $1 \mathrm{mM}$ EGTA, $1 \mathrm{mM} \mathrm{MgCl} 2,0.2 \%$ bovine serum albumin, $\mathrm{pH} 7.2$ ) with successive addition of substrates for Complex I ( $15 \mathrm{mM}$ sodium pyruvate, $15 \mathrm{mM} \mathrm{L}$ proline), Complex III (15 mM glycerol-3-phospate) and Complex IV (1.5 mM $N, N, N^{\prime}, N^{\prime}$-tetramethyl-p-phenylenediamine (TMPD) and $6 \mathrm{mM}$ ascorbate) in presence of $5 \mathrm{mM}$ ADP. Complex I-, III- and IV- driven respiration was corrected for non-respiratory oxygen consumption using $150 \mathrm{nM}$ rotenone, $60 \mathrm{nM}$ antimycin A and $100 \mathrm{nM}$ potassium cyanide, respectively. The total protein concentration was calculated by the Bradford assay and used to normalize the activity of the mitochondrial complexes.

\subsection{Blue native gel electrophoresis and in-gel activity assay}

To measure activity and formation of respiratory complexes and supercomplexes, isolated mitochondria from 40 to 50 pupae or threeday old adult females of the four selected ORT cybrid strains (haplotypes ORT, KSA2, WT5A and VAG1) were used in blue native gel electrophoresis (BNE) and in-gel activity assays. The samples were homogenized gently at $4{ }^{\circ} \mathrm{C}$ with a chilled mortar and pestle with $600 \mu \mathrm{l}$ of Isolation Buffer (250 mM sucrose, $5 \mathrm{mM}$ Tris-HCl, $2 \mathrm{mM}$ EGTA, 0.1\% bovine serum albumin, $\mathrm{pH}$ 7.4). The homogenate was filtered through muslin and centrifuged for $3 \mathrm{~min}$ at $200 \times \mathrm{g}_{\max }$. The supernatant was then centrifuged at $9000 \times g_{\max }$ for $5 \mathrm{~min}$ to collect mitochondria. The pellet was suspended in $200 \mu \mathrm{l}$ of Isolation Buffer without bovine serum albumin. The protein concentration of the resulting mitochondrial preparation was measured using the Bradford assay and the samples were stored at $-80^{\circ} \mathrm{C}$ prior to analysis. BNE, used to separate respiratory complexes in their active native conformation, was performed using the
NativePAGE Novex Bis-Tris gel System (Invitrogen Life Technologies). Aliquots of $100 \mu \mathrm{g}$ of mitochondria were dissolved in $25 \mu \mathrm{l}$ of $1 \times$ Native PAGE Sample Buffer, pH 7.2, containing 50 mM BisTris- 6 N HCl, 50 mM $\mathrm{NaCl}, 10 \% \mathrm{w} / \mathrm{v}$ glycerol, $0.001 \%$ Ponceau $\mathrm{S}$, in the presence of $1 \%$ digitonin and EDTA-free protease inhibitors (Roche), incubated $15 \mathrm{~min}$ on ice, and then centrifuged for $30 \mathrm{~min}$ at $16,000 \times g_{\max }$ at $4{ }^{\circ} \mathrm{C}$. The supernatant containing the inner membrane complexes was mixed with $4 \times$ Native PAGE Sample Buffer, 5\% Coomassie G-250 in a total volume of $40 \mu \mathrm{l} .30 \mu \mathrm{g}$ of protein was loaded onto the NativePAGE Novex Bis-Tris gel and electrophoresis was performed at $70 \mathrm{~V}$ for $1 \mathrm{~h}$ in Anode Buffer containing $50 \mathrm{mM}$ Bis-Tris, $50 \mathrm{mM}$ Tricine, $\mathrm{pH} 6.8$ and Dark Blue Cathode Buffer containing $50 \mathrm{mM}$ Bis-Tris, $50 \mathrm{mM}$ Tricine and $0.02 \%$ Coomassie G-250 at $4{ }^{\circ} \mathrm{C}$. The Dark Blue Cathode Buffer was replaced by Light Blue Buffer containing 0.002\% Coomassie G-250 and the electrophoresis was continued for $21 \mathrm{~h}$ at $25 \mathrm{~V}$. The in-gel activity staining for Complexes I and IV was performed by incubating the gels for $24 \mathrm{~h}$ at room temperature with a solution containing $2 \mathrm{mM}$ Tris- $\mathrm{HCl}$, $\mathrm{pH} 7.4,0.1 \mathrm{mg} / \mathrm{ml} \mathrm{NADH}$ and $2.5 \mathrm{mg} / \mathrm{ml}$ nitrotetrazolium blue (Sigma) for Complex I activity, and with a solution of $0.05 \mathrm{M}$ phosphate buffer pH 7.4 (9 ml) containing $5 \mathrm{mg}$ 3.3'-diamidobenzidine tetrahydrochloride, $1 \mathrm{nM}$ catalase, $10 \mathrm{mg}$ cytochrome $C$ and $750 \mathrm{mg}$ sucrose for Complex IV activity. Gels were washed in distilled water and scanned using an Epson Perfection V750 Pro scanner.

\subsection{Statistics}

mtDNA copy number variation across flies of different haplotype, sex and age groups was analyzed via multi- or one-way analyses of variance (ANOVA), followed by the Tukey post hoc test of significance, as indicated in the figure legends. Multiple linear regressions were used to perform correlation analyses among biochemical and phenotypical data, using the calculated average of these parameters, and the obtained $\mathrm{R}^{2}$ was tested for significance via ANOVA, as indicated in the figure legends. All statistical analyses were performed using the software SAS University Edition v9.4 (SAS Institute Inc.).

\subsection{Data availability}

Strains are available upon request. Sequence data are available in GenBank (accession numbers are listed in Table S1).

\section{Results}

\section{1. mtDNA polymorphisms show both haplogroup- and haplotype-specific variation}

To begin to evaluate the potential effects of mtDNA sequence variation on diverse aspects of mitochondrial function, we sequenced the complete coding regions of ten strains of $D$. melanogaster that originated from various geographical locations (Table S1). Two mtDNA haplogroups were established based on a maximum likelihood analysis (Fig. 1A). Ninety polymorphic sites were identified within the coding sequences, and 33 of these were haplogroup-specific (Table S2). Alignment of the deduced amino acid sequences identified 17 replacements among the 13 mtDNA encoded OXPHOS genes, with only three of these (ND3, ND4L and ATP8) being completely conserved across all lineages (Table 1 ). Considerable polymorphisms were also found among the two mitochondrial rRNA genes, $12 S$ and $16 S$, but only a single nucleotide change in the $t R N A^{G l u}$ gene was observed in the 22 mitochondrial tRNA genes (Table S2).

We also investigated nucleotide and length variation in the $A+T$ region of the D. melanogaster mitochondrial genome (Lewis et al., 1994). Gel electrophoresis of long-PCR fragments showed that the A+T region varies in size from $\sim 4.5 \mathrm{~kb}$ to $\sim 5.5 \mathrm{~kb}$ among all strains (Fig. 1B), and partial Pacl restriction endonuclease digestion analyses indicated that the number of repeats in the repeat I region varied from three to six 

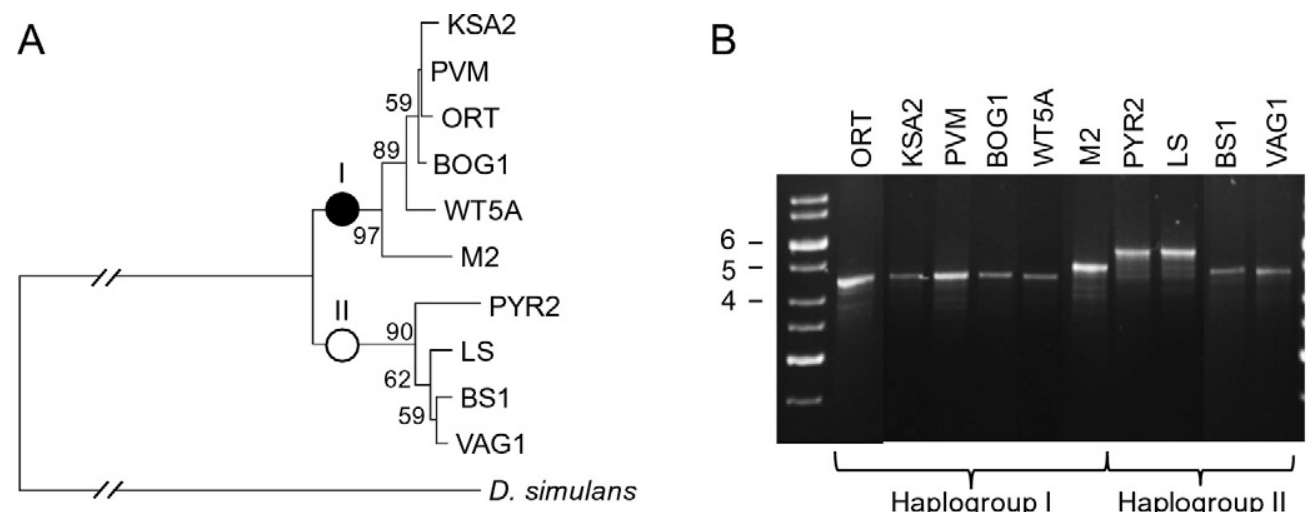

C

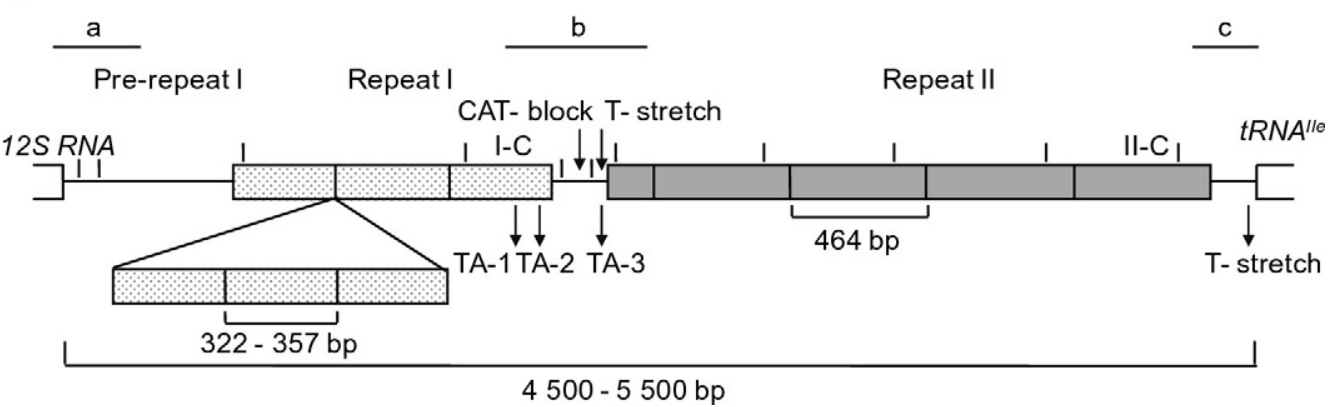

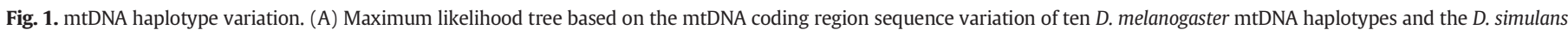

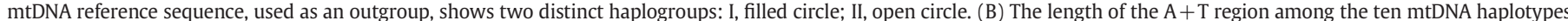

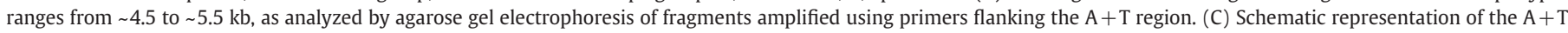

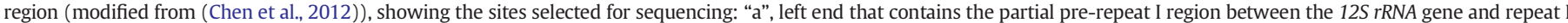

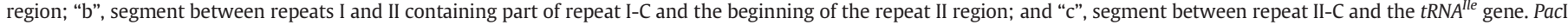
sites are indicated by short vertical lines.

among the mtDNA haplotypes (Fig. S1), accounting for most of the length differences among these mitochondrial genomes. Most haplotypes carried four copies of repeat I, but ORT had three, M2 had five, and LS and PYR2 had six. The repeat II region did not show size variation among the mtDNA haplotypes analyzed here (data not shown). We also sequenced 1425 nucleotides from selected sections of the $\mathrm{A}+\mathrm{T}$ regions (divided into three segments, labeled as a-c in Fig. 1C) and found both haplogroup- and haplotype-specific SNPs throughout the sequences, and length variation within the TA-1, -2 and -3 repeat blocks, CATblock, and middle and right end T-stretches (Fig. 1C, Fig. S2, Table S3). Though there was substantial variation in the $A+T$ region, only the number of repeat I copies and the total length of the mtDNA genome are correlated statistically with the other parameters examined among the ten strains, as described in the following sections.

\subsection{Naturally varying $m t D N A$ haplotypes influence mtDNA copy number}

We evaluated the effects of the ten mtDNA haplotypes on relative mtDNA copy number of four adult age groups by comparing copy numbers in their original nuclear backgrounds, and as cybrids using the ORT nuclear background. First, we observed no significant differences when the strains were grouped into haplogroups, of either sex, whether in the original or the ORT nuclear background (Fig. 2). Although variation was greater among the strains with the mtDNAs in their original nuclear backgrounds, a clear trend was observed for almost all haplotypes, regardless of nuclear background: whereas female mtDNA copy number was significantly higher at ages three and ten days, male copy number was generally more uniform throughout the first 25 days of adult life (Fig. 2, S3 and S4). Interestingly, we found that higher mtDNA copy

Table 1

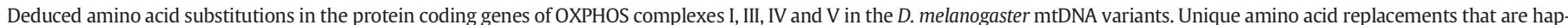
lotype-specific are indicated in bold.

\begin{tabular}{|c|c|c|c|c|c|c|c|c|c|c|c|c|c|c|}
\hline \multirow[t]{2}{*}{ mtDNA } & \multirow[t]{2}{*}{ mtDNA haplogroup } & \multicolumn{7}{|l|}{ I } & \multirow{2}{*}{$\begin{array}{l}\text { III } \\
\text { CYTB }\end{array}$} & \multicolumn{3}{|l|}{ IV } & \multicolumn{2}{|l|}{ V } \\
\hline & & ND1 & ND2 & ND3 & ND4 & $N D 4 L$ & ND5 & ND6 & & $\mathrm{COI}$ & COII & COIII & ATP6 & ATP8 \\
\hline ORT & I & $\mathrm{V}_{190} \mathrm{M}$ & $\mathrm{I}_{277} \mathrm{~L}$ & & & & $\mathrm{M}_{502} \mathrm{I}$ & & & & & & $\mathrm{S}_{538} \mathrm{P}, \mathrm{M}_{559} \mathrm{~V}$ & \\
\hline KSA2 & I & $\mathrm{V}_{190} \mathrm{M}$ & $\mathrm{I}_{277} \mathrm{~L}$ & & & & $\mathrm{M}_{502} \mathrm{I}$ & & $\mathrm{D}_{21} \mathrm{~N}$ & & & $\mathbf{A}_{75} \mathbf{T}$ & $\mathrm{S}_{538} \mathrm{P}, \mathrm{M}_{559} \mathrm{~V}$ & \\
\hline PVM & I & $\mathrm{V}_{190} \mathrm{M}$ & $\mathrm{I}_{277} \mathrm{~L}$ & & & & $\mathrm{M}_{502} \mathrm{I}$ & & & & & & $\mathrm{S}_{538} \mathrm{P}, \mathrm{M}_{559} \mathrm{~V}$ & \\
\hline BOG1 & I & $\mathrm{V}_{190} \mathrm{M}$ & $\mathrm{I}_{277} \mathrm{~L}$ & & & & $\mathbf{A}_{\mathbf{3 5 6}} \mathrm{T}, \mathrm{M}_{502} \mathrm{I}$ & & & & & & $\mathrm{S}_{538} \mathrm{P}, \mathrm{M}_{559} \mathrm{~V}$ & \\
\hline WT5A & I & $\mathrm{V}_{190} \mathrm{M}$ & $\mathrm{I}_{277} \mathrm{~L}$ & & & & $\mathrm{M}_{502} \mathrm{I}$ & & & & & & $\mathrm{S}_{538} \mathrm{P}, \mathrm{M}_{559} \mathrm{~V}$ & \\
\hline M2 & I & $\mathrm{V}_{190} \mathrm{M}$ & $\mathrm{I}_{277} \mathrm{~L}, \mathbf{M}_{\mathbf{2 8 0}} \mathbf{V}$ & & $\mathrm{V}_{161} \mathrm{~L}$ & & $\mathrm{M}_{502} \mathrm{I}$ & & & $A_{106} T$ & & & $\mathbf{M}_{185} \mathbf{I}, \mathrm{S}_{538} \mathrm{P}, \mathrm{M}_{559} \mathrm{~V}$ & \\
\hline PYR2 & II & & & & & & & $N_{114} D$ & $\mathrm{~V}_{264} M$ & & & & & \\
\hline LS & II & & & & & & $S_{59} F$ & & & & & $D_{13} N$ & & \\
\hline BS1 & II & & & & & & & & & & $\mathrm{G}_{58} \mathrm{~S}$ & & & \\
\hline VAG1 & II & & & & & & & & & & & & & \\
\hline
\end{tabular}



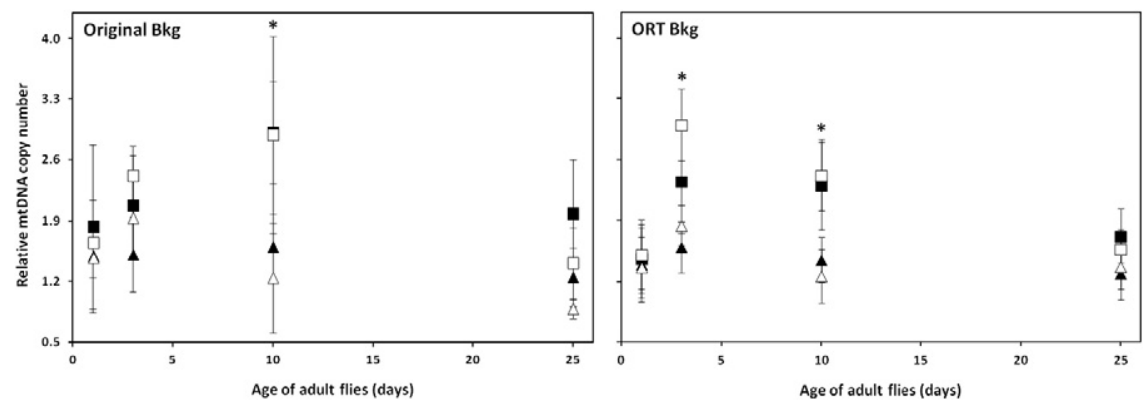

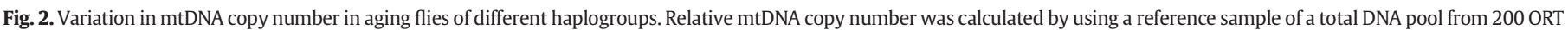

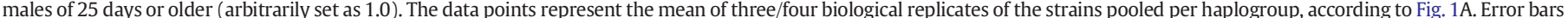

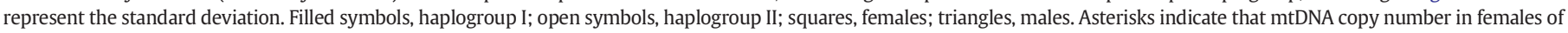

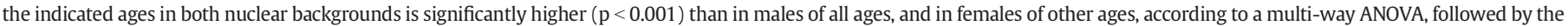
Tukey post hoc test. Bkg, nuclear background.

number among the original strains in both sexes is associated statistically with $\mathrm{A}+\mathrm{T}$ regions bearing five or six repeat I copies (Fig. 3). In the ORT cybrids, mtDNA copy number remained low when the $A+T$ region had only three repeat I copies (especially in females at age three days), whereas no significant differences were observed for haplotypes with four, five or six copies. The ORT haplotype, the only one with three repeat I copies, also presented significantly lower mtDNA copy number in females of age three days when tested as a cybrid using the M2 nuclear background (Fig. 4), showing that low mtDNA copy number can be maintained in a mtDNA haplotype with only three copies of repeat I, despite the possible disruption of mito-nuclear genome interactions. In summary, our data do not reveal a haplogroup-specific contribution to mtDNA copy number among the strains analyzed, but do indicate an influence of the number of repeat I copies in the $A+T$ region of the given haplotype.

We evaluated the effects of ORT, another low (KSA2) and two high (WT5A and VAG1) copy number haplotypes (based on the ORT cybrid data, Figs. S3 and S4) on mtDNA copy number in the M2 nuclear background. We found that female mtDNA copy number at its highest levels (age three days) was similar in the ORT and M2 nuclear backgrounds (Fig. 4A), suggesting that it is determined by the mtDNA haplotypes themselves. In analyzing the two unique amino acid replacements in
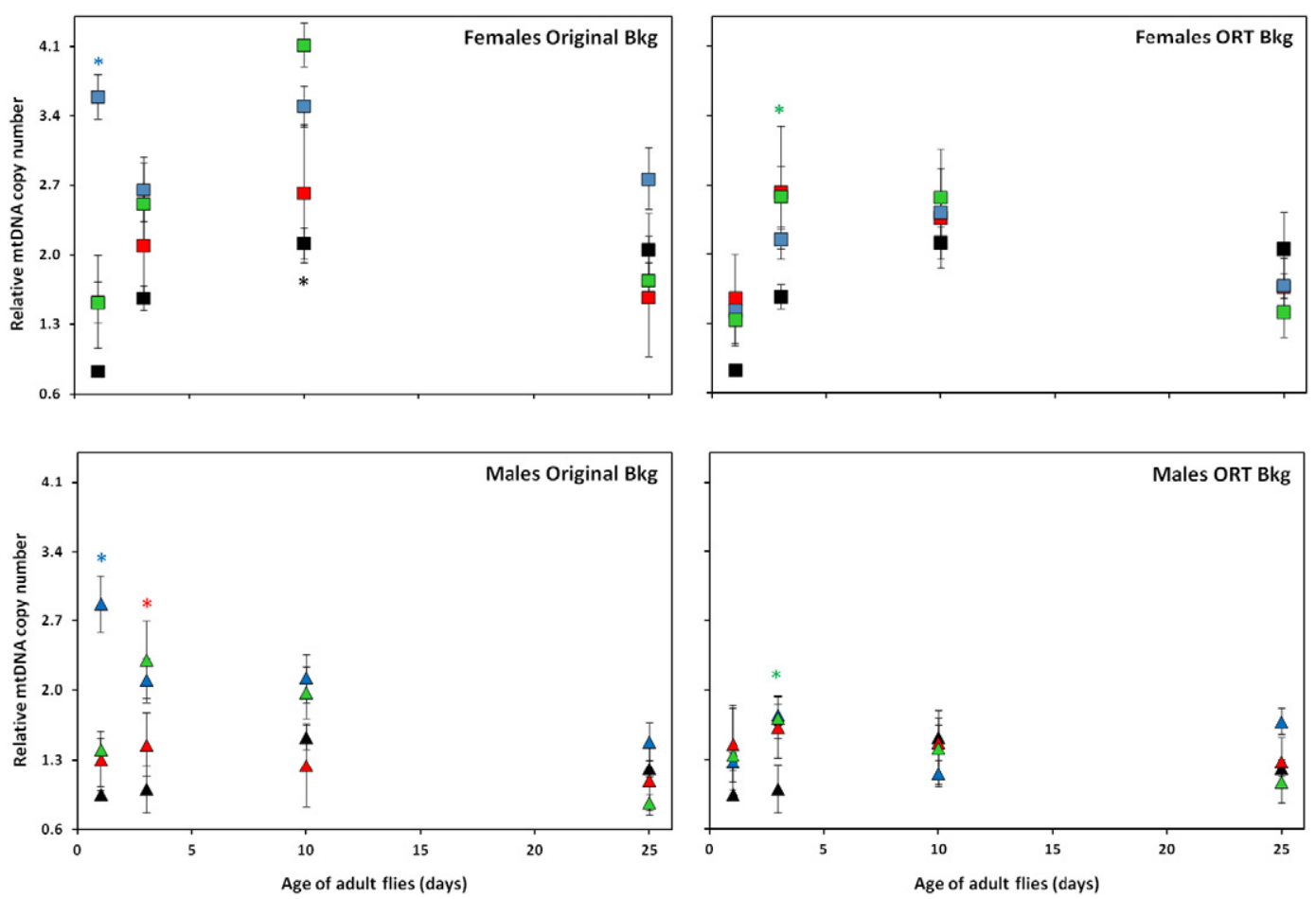

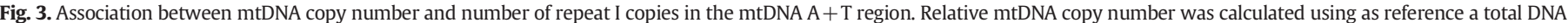

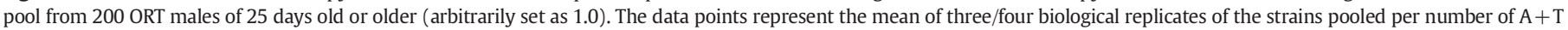

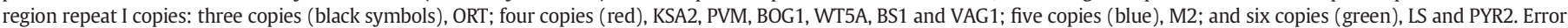

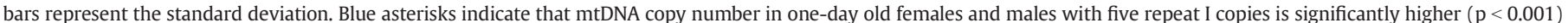

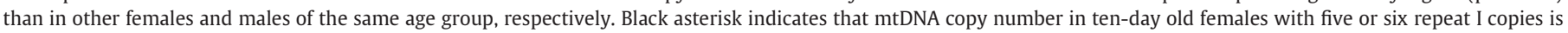

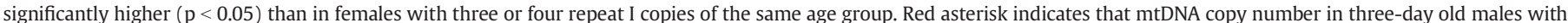

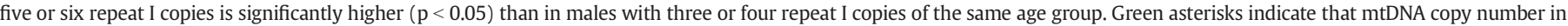

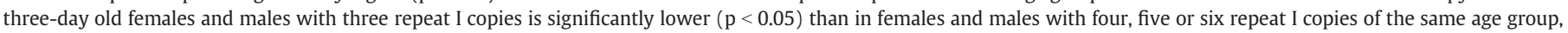
respectively. The statistical analyses were performed via multi-way ANOVA, followed by the Tukey post hoc test. 
A

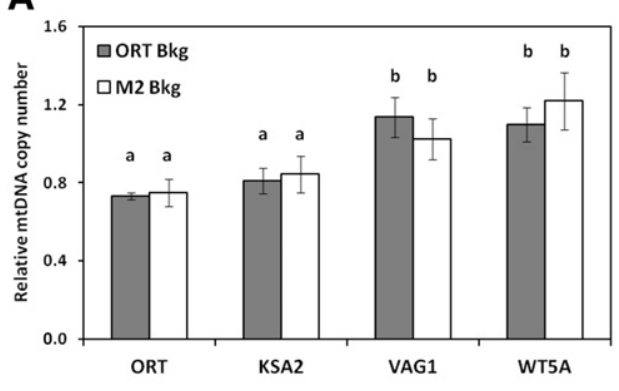

B

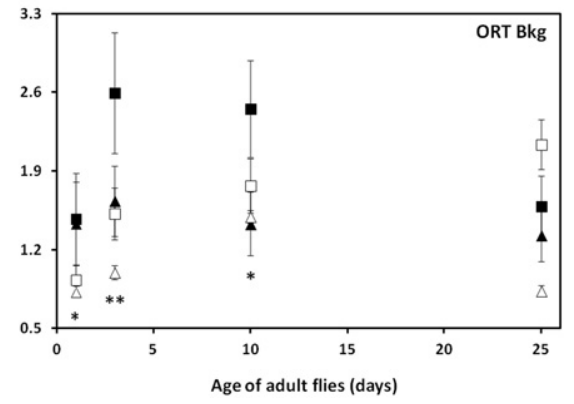

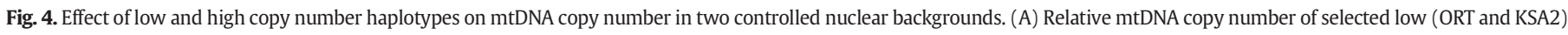

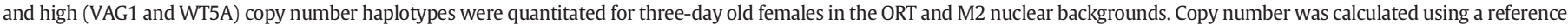

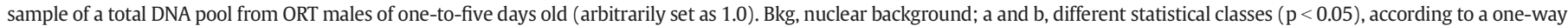

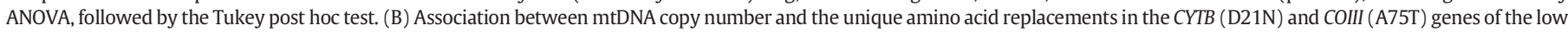

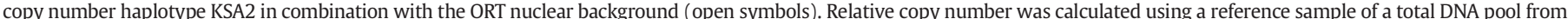

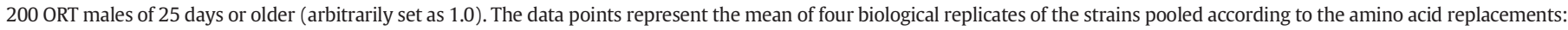

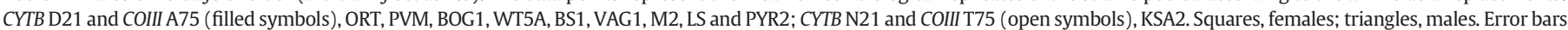

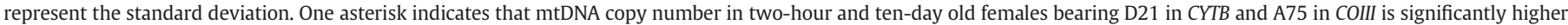

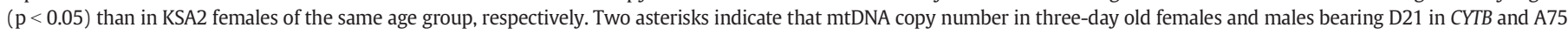

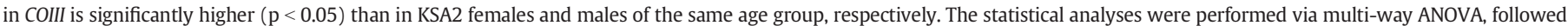
by the Tukey post hoc test.

the CYTB (D21N) and COIII (A75T) genes of the KSA2 haplotype, we also found a positive correlation with low mtDNA copy number in the ORT nuclear background (Fig. 4B).

\subsection{Interactions between mtDNA and nuclear background influence development}

The effect of mtDNA haplotypes on phenotypic traits was examined by evaluation of egg-to-adult development time and juvenile body weight. As with mtDNA copy number, we observed that variation among haplotypes was highest in their original nuclear background, compared with the ORT and M2 cybrid strains (Figs. S5 and S6). We found positive correlations between development time and mtDNA copy number, and between body weight and mtDNA copy number in females of the original nuclear background strains (Fig. 5). $>90 \%$ of this variation is attributable to variation in mtDNA copy number. Interestingly, the correlation between body weight and mtDNA copy number was lost in female ORT cybrids, but that between development time and mtDNA copy number remained strong (Fig. 5B). No correlation was observed between mtDNA copy number and development time or body weight in males (data not shown). However, approximately $70 \%$ of the variation in development time in males can be explained by the variation in mtDNA copy number in females, in both the original and the ORT nuclear backgrounds (Fig. 5B). In the ORT nuclear background, the variation in body weight in males was also correlated with female mtDNA copy number $\left(\mathrm{R}^{2}=0.41, \mathrm{p}=0.045\right.$; data not shown).

Conversely, we found a significant and positive correlation between body weight in females and mtDNA copy number in males, in the original nuclear background strains, although not in the ORT cybrid strains. This accounted for almost $70 \%$ of the variation in female body weight (Fig. 5A). Taken together, our data suggest that mtDNA copy number (regardless of sex) influences female body weight but this relationship is dependent on mito-nuclear genome interactions. In agreement with this proposition, we found no statistical correlations between female body weight and mtDNA copy number in the M2 cybrid strains (data not shown).

As regards developmental time, this was strongly correlated in both sexes with mtDNA copy number in females in the ORT nuclear background, especially at the age when the females are most fertile (3- to 10 days). Put simply, high mtDNA copy number is correlated with longer development time. Although not statistically significant, we observed an opposite trend in the M2 cybrids: the more mtDNA in three-day old females, the faster the development $\left(R^{2}=0.36\right.$ for females, and 0.83 for males), again suggesting the importance of mito-nuclear genome interactions.

\section{4. mtDNA variation affects OXPHOS parameters}

To evaluate the relationship between mtDNA sequence variation, copy number, and phenotype, we measured mitochondrial substrate oxidation by OXPHOS Complexes I, III and IV, as well as the formation and activity of SCs containing Complex I and IV, in 3 day-old adults, using ORT cybrids with both high (VAG1, WT5A) and low copy number (ORT, KSA2). Both of the low copy number haplotypes showed elevated oxidation of Complex I- and III-linked substrates whereas Complex IVdriven oxygen consumption was uniform among the cybrids tested (Fig. 6A).

To investigate the molecular nature of these effects, we used BNE combined with in-gel histochemistry for Complexes I and IV. SCs manifesting Complex I activity were elevated (Fig. 6B), implying that the higher rates of Complex I-linked substrate oxidation observed in these cybrids are linked to a higher order arrangement of the OXPHOS complexes in the ORT and KSA2 haplotypes. At the same time, both the ingel activity of Complex IV and its presence in SCs were uniform among the four strains (Fig. 6B).

Although not supported statistically because of the low sampling, we did find an important correlation between Complex I- and III-linked substrate oxidation and the phenotypic parameters associated with low mtDNA copy-number, i.e., developmental time, and female body weight (Fig. S8). Our data suggest that higher OXPHOS activity associated with increased SCs may be the biochemical explanation for the faster development and decreased body weight in females, in the lines with lower mtDNA copy number. Because these phenotypic parameters are dependent on development itself, we also examined mtDNA copy number, substrate oxidation and SC representation in the selected low and high copy number ORT cybrids at the pupal stage, but found no significant differences among the four lines (Fig. S7C-E).

\section{Discussion}

In this report, we have demonstrated effects of mtDNA sequence variation, both in the coding and non-coding $\mathrm{A}+\mathrm{T}$ regions, on mtDNA copy number, and found correlations between mtDNA copy number, developmental traits, and mitochondrial biochemistry. Naturally occurring mtDNA variants have been shown previously to affect 
A

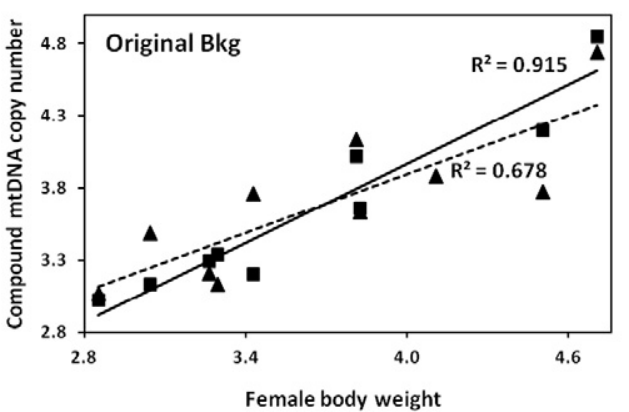

B
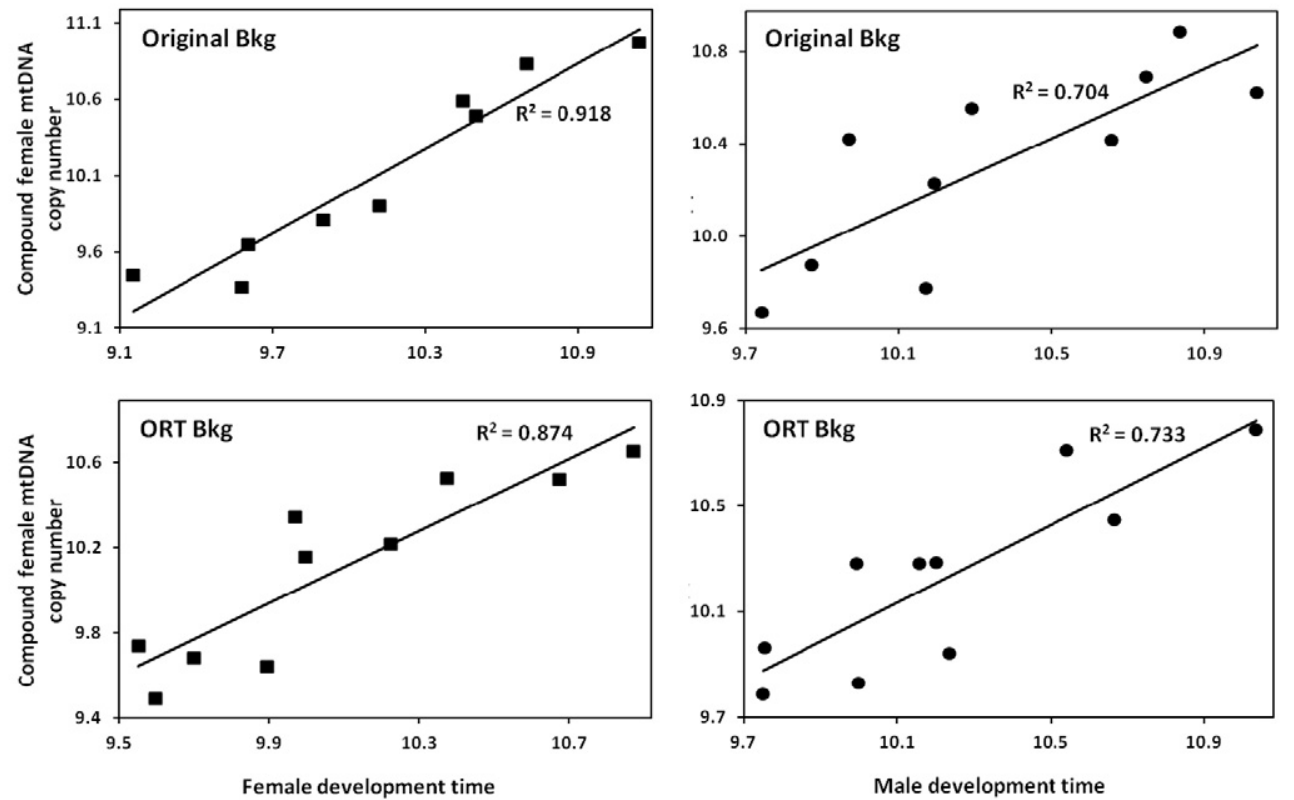

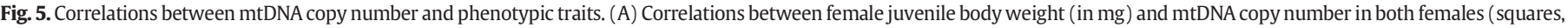

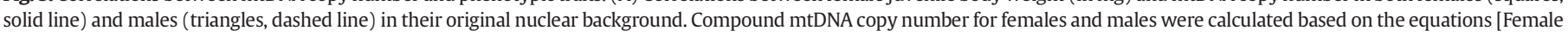

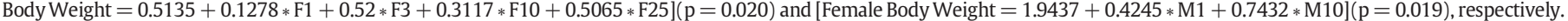

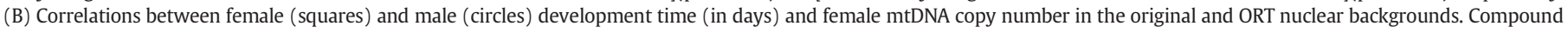

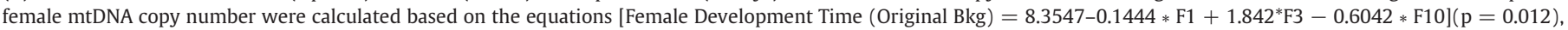

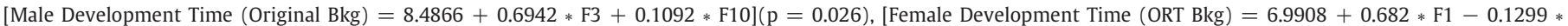

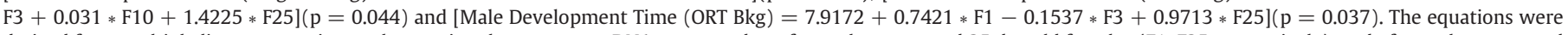

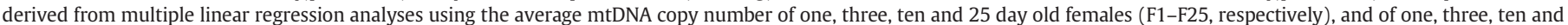
25 day old males (M1-M25, respectively). The p values represent the significance of the $\mathrm{R}^{2}$ shown on the graphs, obtained by ANOVA.

mitochondrial bioenergetics in D. melanogaster (Aw et al., 2011; Innocenti et al., 2011; Clancy, 2008), D. simulans (Katewa and Ballard, 2007; Melvin and Ballard, 2006; Pichaud et al., 2010), mice (MorenoLoshuertos et al., 2006) and humans (Amo and Brand, 2007; Elson et al., 2007). It has also been shown that mtDNA variation affects mtDNA copy number and gene expression, and that this can be linked to sexspecific effects on fertility and longevity in Drosophila (Camus et al., 2015). mtDNA variation in humans has been connected to susceptibility to complex disorders, and to tissue-specific alterations in mitochondrial activity (Moreno-Loshuertos et al., 2006; Kazuno et al., 2006).

Among the 13 proteins encoded in mtDNA, we found several haplotype-specific amino acid replacement variants, eight of which (ND5 S59F in LS, ND5 A356T in BOG1, ND6 N114D in PYR2, CYTB D21N in KSA2, COI A106T in M2, COII G58S in BS1, COIII D13N in LS, and COIII A75T in KSA2) are judged more likely to have functional consequences due to their non-conservative nature. However, only CYTB D21N and/or COIII A75T, both specific to haplotype KSA2, were associated with low mtDNA copy number in controlled nuclear backgrounds. The extent to which these amino acid changes alter the structure and function of the OXPHOS proteins, causing an impact on copy number, remains to be evaluated thoroughly. We also found that low mtDNA copy number in the ORT haplotype is associated with a low number of copies of the $\mathrm{A}+\mathrm{T}$ repeat I element. Though mtDNA coding region variation has been studied more extensively, variation in the A + T region in Drosophila has attracted less attention, despite the potential for size and sequence variation in this region to influence mtDNA replication and/or transcription. A specific replication origin has been shown to lie within the A + T region of Drosophila mtDNA (Jõers and Jacobs, 2013; Clary and Wolstenholme, 1987). We speculate that lower representation of the repeat I element could decrease the frequency of mtDNA replication initiation, leading to a lower mtDNA copy number. One might expect that replication and transcription of shorter mtDNA variants, as is the case for the ORT haplotype, is completed faster, so there might be a compensatory process to keep a lower steady-state number of mtDNA molecules in the cells to balance transcription and/or translation inside mitochondria. However, how this would influence SC formation and the efficiency of OXPHOS is unclear.

mtDNA variation and its effects on fitness and adaptation have been observed in humans, mice, hares, finches, copepods and Drosophila (Meiklejohn et al., 2007; Dowling et al., 2008; Galtier et al., 2009; 
A
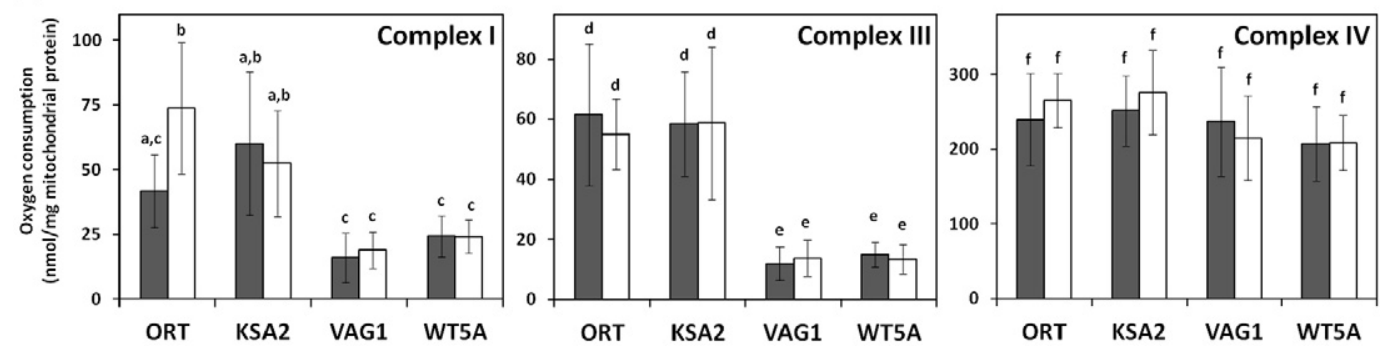

B
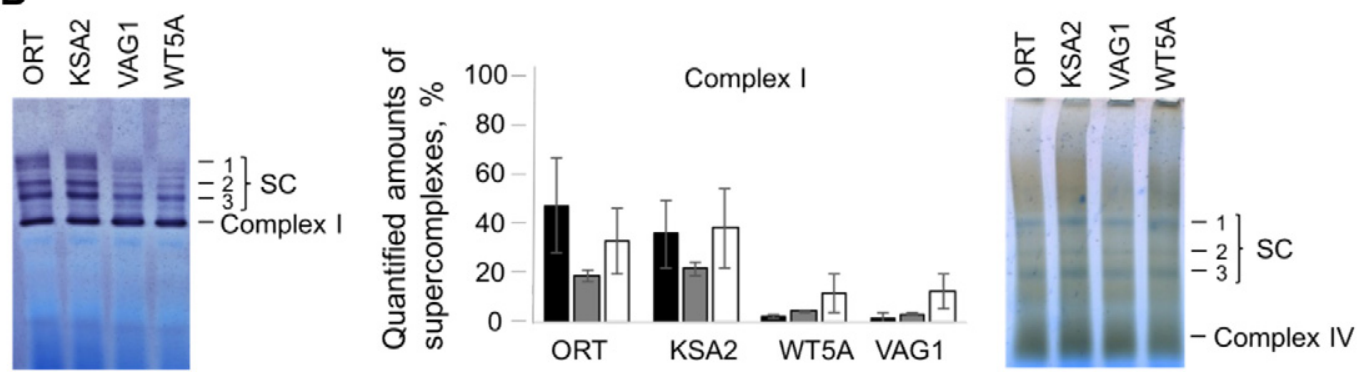

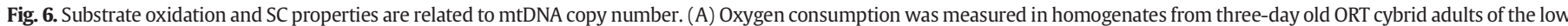

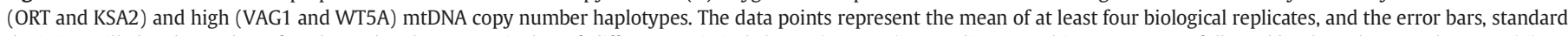

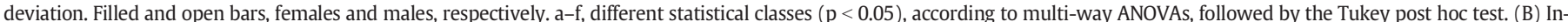

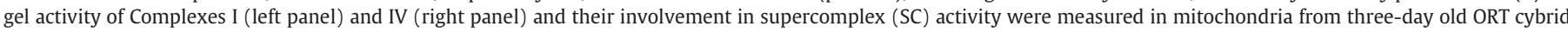

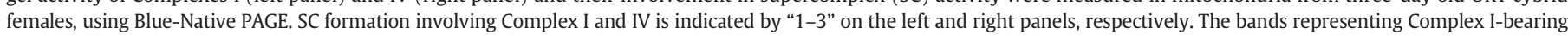

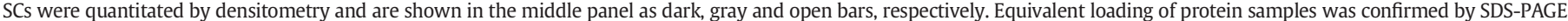
stained with Coomassie blue (Fig. S7B).

Ballard and Whitlock, 2004; William and Ballard, 2005; Ballard and Melvin, 2010). Selective pressure on human mtDNA variants has been suggested to lead to mtDNA-specific adaptations to different environments (Blier et al., 2001; Fontanillas et al., 2005; Wallace, 2005). Using controlled nuclear backgrounds via the construction of cybrid strains has revealed the importance of mito-nuclear interactions among different geographically-isolated populations of $D$. simulans and the marine copepod Tigriopus californicus. These studies showed that disrupting these interactions in allopatric populations can lead to a decrease in OXPHOS activities, including Complex IV (Edmands and Burton, 1998; Sackton et al., 2003), Complexes I and III, and ATP production (Ellison and Burton, 2006). Other studies have also illustrated the effects of disrupting co-evolved mito-nuclear allelic complexes on aging (Clancy, 2008; Rand et al., 2006) and male fertility-related traits (Yee et al., 2013) in Drosophila. Here, we studied two metabolically important phenotypic traits, egg-to-adult development time and juvenile body weight, and showed their positive correlations with mtDNA copy number in females of the original strains. We showed that the correlation between mtDNA copy number and body weight is disrupted in the ORT cybrid strains, suggesting some level of inter-genomic co-adaptation in the wild strains. If the negative effects of disrupting coevolved mito-nuclear gene complexes were to increase with greater evolutionary distance we would have expected haplogroup II cybrids to manifest more substantial phenotypic effects than those from haplogroup I, because a haplogroup I strain was used as the nuclear recipient. This was clearly not the case in our study. It was shown earlier that introduction of $D$. simulans mtDNA into a $D$. melanogaster nuclear background does not result in performance that differs significantly from that of native D. melanogaster mtDNA (Rand et al., 2006; Montooth et al., 2010). Either our assumption is false in this case, or ORT represents a strain into which haplogroup I mtDNA has introgressed in the wild.

The correlations we identified between development time and mtDNA copy number were as strong in the original strains as in the corresponding ORT cybrids, suggesting that mtDNA copy number is either a determinant or a strong marker of this phenotypic trait. Because mtDNA copy number was significantly higher in females during the main period of reproductive activity (three to ten days after eclosion), we attribute this phenomenon to the mtDNA levels in the female ovaries, which are known to have higher mtDNA content than other organs (Wolff et al., 2013). Interestingly, it has been shown that a longer development time in Drosophila, which in our case is associated with more mtDNA in females at reproductive age, has a competitive disadvantage with a direct impact in the ecology of the organism, as the individuals that develop faster can outcompete the slower ones for the limited food source during the larval stages (De Miranda and Eggleston, 1988a, 1988b, 1988c).

We also document here that the supposedly advantageous faster development time, associated with lower levels of mtDNA, is also associated with and may even be caused by higher rates of mitochondrial oxygen consumption and OXPHOS SC formation in the ORT nuclear background. To achieve optimal ATP production during development, one might expect a dynamic balance between the free OXPHOS complexes and their SCs within the inner mitochondrial membrane (AcinPerez and Enriquez, 2014). Higher order arrangement of the OXPHOS complexes may channel electron transport, increasing mitochondrial metabolism and influencing the organism's time of development. However, it remains unclear how lower mtDNA copy number, which we report can be associated with a low number of $A+T$ repeat I elements (as in the ORT haplotype) or specific amino acid substitutions in CYTB and/ or COIII genes (as in the KSA2 haplotype), may lead to higher respiration rates via enhanced SC formation. We speculate that a particular retrograde signal from "healthy" low copy-number mitochondria promotes a nuclear response for more efficient electron transport via mitochondrial import of SC assembly factors, to compensate for the supposedly lower expression of mitochondrial genes, but this has yet to be tested experimentally. Another possibility may be that non-optimal high copy number results in an excess production of one particular OXPHOS complex which destabilizes, or at least renders stoichiometrically suboptimal, the overall structure of the respiratory membrane.

Rapid development is associated with lower adult weight (Ashburner, 1989). Our data show strong positive correlation between mtDNA copy number in both sexes with female body weight, although 
this is only true for the original strains. In D. melanogaster, several studies have shown positive correlations between body weight and both male reproductive success, and female fecundity (Markow, 1988; Parsons, 1974). Low mtDNA copy number may therefore come with a trade-off: the animals may develop faster and outcompete more slowly developing individuals with high mtDNA copy number; but the resulting, smaller adults will not be as successful reproductively.

Our dataset also allowed us to test the hypothesis that phenotypic variation caused by higher mtDNA mutation load should be more evident in males, because mitochondrial function is only under adaptive selection in females (Frank and Hurst, 1996). In agreement with recent findings (Mossman et al., 2016), our data do not support this prediction. We found that females were as sensitive as males to mtDNA variation in traits that are known to be sexually dimorphic, such as developmental time and body weight.

In summary, our data support the idea that mtDNA copy number is largely under mitochondrial genetic control, and that this influences organismal phenotype by virtue of resulting effects on the organization of the respiratory membrane and the efficiency of OXPHOS. How our findings relate to fly models of mitochondrial disease will be an important area of research to pursue, because most of these models manifest development problems and mtDNA copy number alterations that are believed to mimic mitochondrial disorders in humans.

\section{Author contributions}

TSS, MTO, GC and PL performed and analyzed the experiments; TSS, MTO, HTJ and LSK wrote the manuscript; HTJ and LSK conceived of and supervised the research. The authors declare no competing financial interests.

\section{Acknowledgements}

We thank Jani Haukka and Tess Jeffers for their contributions in the analyses of mtDNA sequences, and Charlotta Putkonen and Troy Faithfull for technical assistance. This work was supported by grants from the Academy of Finland (FiDiPro and FinMIT Centre of Excellence grants to H.T.J.) and the National Institutes of Health (GM45295 to L.S.K.). M.T.O. acknowledges funding from the Marie Curie International Incoming Fellowship Program (grant 328988) and the Fundação de Amparo à Pesquisa do Estado de São Paulo (grant 2014/02253-6).

\section{Appendix A. Supplementary data}

Supplementary data to this article can be found online at http://dx. doi.org/10.1016/j.mito.2017.02.001.

\section{References}

Acín-Pérez, R., Enriquez, J.A., 2014. Biochim. Biophys. Acta Bioenerg. 1837, 444-450. Acín-Pérez, R., Fernández-Silva, P., Peleato, M.L., Pérez-Martos, A., Enriquez, J.A., 2008. Mol. Cell 32, 529-539.
Amo, T., Brand, M.D., 2007. Biochem. J. 404, 345-351.

Ashburner, M., 1989. Drosophila. A Laboratory Handbook. Cold Spring Harbor Laboratory Press.

Aw, W.C., Correa, C.C., Clancy, D.J., Ballard, J.W.O., 2011. Mitochondrion 11, 756-763.

Ballard, J.W.O., Melvin, R.G., 2010. Mol. Ecol. 19, 1523-1539.

Ballard, J.W.O., Whitlock, M.C., 2004. Mol. Ecol. 13, 729-744.

Ballard, J.W.O., Melvin, R.G., Katewa, S.D., Maas, K., 2007. Evolution (N. Y). 61, 1735-1747.

Blier, P.U., Dufresne, F., Burton, R.S., 2001. Trends Genet. 17, 400-406.

Bogenhagen, D., Clayton, D.A., 1977. Cell 11, 719-727.

Camus, M.F., Wolf, J.B.W., Morrow, E.H., Dowling, D.K., 2015. Curr. Biol. 25, 2717-2722.

Chaban, Y., Boekema, E.J., Dudkina, N.V., 2014. Biochim. Biophys. Acta Bioenerg. 1837, 418-426.

Chen, S., Oliveira, M.T., Sanz, A., Kemppainen, E., Fukuoh, A., Schlicht, B., Kaguni, L.S., Jacobs, H.T., 2012. Genetics 192, 483-493.

Clancy, D.J., 2008. Aging Cell 7, 795-804.

Clary, D.O., Wolstenholme, D.R., 1987. J. Mol. Evol. 25, 116-125.

Cogliati, S., Acín-Pérez, R., Enriquez, J.A., 2016. Redox Proteins in Supercomplexes and Signalosomes Chapter 3. CRC Press.

De Miranda, J.R., Eggleston, P., 1988a. Heredity. 60, 213-219.

De Miranda, J.R., Eggleston, P., 1988b. Heredity. 60, 205-212.

De Miranda, J.R., Eggleston, P., 1988c. Heredity. 61, 339-346.

Dowling, D.K., Chavez, K.A., Arnqvist, G., 2007. Evolution 61, 194-201.

Dowling, D.K., Friberg, U., Lindell, J., 2008. Trends Ecol. Evol. 23, 546-554.

Edmands, S., Burton, R.S., 1998. Heredity. 80, 668-674.

Ellison, C.K., Burton, R.S., 2006. Evolution. 60, 1382-1391.

Elson, J.L., Turnbull, D.M., Taylor, R.W., 2007. Biochem. J. 404 (2), e3.

Fauron, C.M., Wolstenholme, D.R., 1976. Proc. Natl. Acad. Sci. 73, 3623-3627.

Fontanillas, P., Dépraz, A., Giorgi, M.S., Perrin, N., 2005. Mol. Ecol. 14, 661-670.

Frank, S.A., Hurst, L.D., 1996. Nature 383, 224.

Galtier, N., Nabholz, B., GlÉmin, S., Hurst, G.D.D., 2009. Mol. Ecol. 18, 4541-4550.

Goddard, J.M., Wolstenholme, D.R., 1978. Proc. Natl. Acad. Sci. 75, 3886-3890.

Gordon, D., Abajian, C., Green, P., 1998. Genome Res. 8, 195-202.

Hutter, C.M., Rand, D.M., 1995. Genetics 140, 537-548.

Innocenti, P., Morrow, E.H., Dowling, D.K., 2011. Science 332, 845-848.

James, A.C., Ballard, J.W.O., 2003. Genetics 164, 187-194.

Jobb, G., Haeseler, A., Strimmer, K., 2004. BMC Evol. Biol. 4 (18).

Jõers, P., Jacobs, H.T., 2013. PLoS One 8 (1).

Katewa, S.D., Ballard, J.W.O., 2007. Insect Biochem. Mol. Biol. 37, 213-222.

Kazuno, A., Munakata, K., Nagai, T., Shimozono, S., Tanaka, M., Yoneda, M., Kato, N., Miyawaki, A., Kato, T., 2006. PLoS Genet. 2, e128.

Lewis, D.L., Farr, C.L., Farquhar, A.L., Kaguni, L.S., 1994. Mol. Biol. Evol. 11, 523-538.

Markow, T.A., 1988. J. Comp. Psychol. 102, 169.

Meiklejohn, C.D., Montooth, K.L., Rand, D.M., 2007. Trends Genet. 23, 259-263.

Melvin, R.G., Ballard, J.W.O., 2006. Aging Cell 5, 225-233.

Montooth, K.L., Meiklejohn, C.D., Abt, D.N., Rand, D.M., 2010. Evolution. 64, 3364-3379.

Moreno-Loshuertos, R., Acín-Pérez, R., Fernandez-Silva, P., Movilla, N., Perez-Martos, A., de Cordoba, S.R., Gallardo, M.E., Enriquez, J.A., 2006. Nat. Genet. 38, 1261-1268.

Mossman, J.A., Tross, J.G., Li, N., Wu, Z., Rand, D.M., 2016. Genetics 116.

Parsons, P.A., 1974. Behav. Genet. 4, 395-404.

Pichaud, N., Chatelain, E.H., Ballard, J.W.O., Tanguay, R., Morrow, G., Blier, P.U., 2010. J. Exp. Biol. 213, 1665-1675.

Rand, D.M., Fry, A., Sheldahl, L., 2006. Genetics 172, 329-341.

Rosca, M.G., Vazquez, E.J., Kerner, J., Parland, W., Chandler, M.P., Stanley, W., Sabbah, H.N., Hoppel, C.L., 2008. Cardiovasc. Res. 80, 30-39.

Sackton, T.B., Haney, R.A., Rand, D.M., 2003. Evolution (N. Y) 57, 2315-2325.

Taylor, R.W., Turnbull, D.M., 2005. Nat. Rev. Genet. 6, 389-402.

Toivonen, J.M., O'Dell, K.M.C., Petit, N., Irvine, S.C., Knight, G.K., Lehtonen, M., Longmuir, M., Luoto, K., Touraille, S., Wang, Z., Alziari, S., Shah, Z.H., Jacobs, H.T., 2001. Genetics 159, 241-254.

Wallace, D.C., 2005. Gene 354, 169-180.

William, D.M.R.J., Ballard, O., 2005. Annu. Rev. Ecol. Evol. Syst. 36, 621-642.

Wolff, J.N., Sutovsky, P., Ballard, J.W.O., 2013. Cell Tissue Res. 353, 195-200.

Wolff, J.N., Pichaud, N., Camus, M.F., Côté, G., Blier, P.U., Dowling, D.K., 2016. J. Evol. Biol.

Yee, W.K.W., Sutton, K.L., Dowling, D.K., 2013. Curr. Biol. 23, R55-R56. 